\title{
Artifacts and Skull Stripping: An Application Towards the Preprocessing for Brain Abnormalities Detection from MRI
}

\author{
Sudipta Roy ${ }^{1 *}$, Debnath Bhattacharyya ${ }^{2}$, Samir K Bandyopadhyay ${ }^{3}$ \\ and Tai-Hoon $\mathrm{Kim}^{4}$ \\ ${ }^{1}$ Department of Computer Science and Engineering, U. V. Patel College of \\ Engineering, Ganpat University, Kherva - 384012, Gujarat, India \\ ${ }^{2}$ Department of Computer Science and Engineering, Vignan's Institute of \\ Information Technology, Visakhapatnam 530049, AP, India \\ ${ }^{3}$ Department of Computer Science and Engineering, Calcutta University \\ Technology Campus, JD-2, Sector-III, Salt Lake, Kolkata 98, India \\ ${ }^{4}$ Department of Convergence Security, Sungshin Women's University, 249-1, \\ Dongseon-dong 3-ga, Seoul 136-742, South Korea \\ sudiptaroy01@yahoo.com;debnathb@gmail.com; skb1@vsnl.com; \\ taihoonn@daum.net
}

\begin{abstract}
Improvement in detection and analysis of brain abnormality and normal tissues is an important task in brain image analysis. Diagnosis quality of brain MR of brain images hampered due to the presence of artifacts and skull. Small abnormalities detection hampered due to the presence of skull region of brain. Sometimes artifacts and skull has been treated as an abnormality in automated system and it hamper the intelligence system. Thus in computerized pre-processed method requires as artifacts and skull removal. In this paper, a preprocessing method based on binarization and wavelet decomposition for improvement of brain abnormality detection and diagnosis has been described. Proposed preprocessing makes the image segmentation more accurate and small error.
\end{abstract}

Keywords: Artifacts, skull stripping, preprocessing, MRI of brain, brain abnormalities, segmentation

\section{Introduction}

Automatic segmentation of brain tissues from MRI is a challenging process due to the variation in brain shapes and similarity of intensity values in the brain and non-brain tissues. Artifacts and undesired tissues (skull) as non brain region affects the quality of processing and may lead to automatic diagnostic confusion. The majority of skull stripping treats the brain as a single connected region separated from non-brain tissues by a rim of CSF. In reality even with high resolution T1 weighted MR images, thin connections between the brain and other cranial structure exist in the form of dura and connective tissue lining venous sinuses. In the present day artifacts in MRI are mainly letters that contains the patients information's and image modality. The proposed preprocessing is very simple technique using the combination of binarazation, wavelet decomposition, and computational geometric as major intermediate steps. It tested with large number of MR images and produce good results. In preprocessing method artifacts removal is considered as mandatory and skull removal is optional. Artifacts removal is required for every post-processing technique and skull removal is required for some abnormality detection like edema, tumor, stroke lesions and hemorrhage lesions but 
sometimes for a particular type of hemorrhage we need distance of abnormal region from skull.

A number of automated and semi-automated skull stripping algorithms are available in literature. Skull re-moval using graph cuts [1] relies on graph theoretic image segmentation techniques to position cuts that serve to isolate and remove skull. Region Growing based [2] method for coronal T1-weighted images plans to automati-cally detect two seed regions of the brain and non-brain by using the mask generated by mathematical morpho-logical operations. Then the seed regions were expanded using $2 \mathrm{D}$ region growing algorithm. Another fully automatic brain extraction method [3] using diffusion, run length encoding and region labeling were developed for skull removal in T2 weighted axial MR brain images. Clustering and 2D Region Growing method [4] for detecting the brain boundaries inside the skull was used to join together the clusters and also remove the skull area. Authors are tested on 4 slices only thus method needed to improvement to process all the slices in the dataset. Robex method [5] for skull stripping by using a shape model trained on healthy brains to be relatively insensitive to lesions inside the brain. Their results showed this method was better than Brain Surface Extractor (BSE), Hybrid Watershed Algorithm (HWA) and Brain Extraction Tool (BET). They used T1 weight images taken from Glioblastoma patients.

A method based [6] on histogram analysis and compared the segmentation accuracy between their proposed method and two widely used techniques, namely BSE and BET. Based on this factor, they reported that their proposed method outperforms these methods.

A convolutional deep learning [7] architecture is used to skull removal but not limited to non-enhanced T1 images. When trained appropriately, it handles an arbitrary number of modalities including contrast-enhanced scans. Its applicability to MRI data, comprising four channels: non-enhanced and contrast-enhanced T1w, T2w and FLAIR contrasts, is demonstrated on a challenging clinical data set containing brain tumors. A mathematical morphology method was implemented [8] for the preprocessing of MR brain images for the improved segmentation of brain tumor based on mathematical morphology operations. The first part of this paper [8] was an efficient method for the skull stripping of brain MR images based on mathematical morphology. In that paper brain part was identified by the largest connected component in the image after binarization. The largest connected component is then dilated with a $3 \times 3$ square structuring element so as to preserve minute brain information in the output image. The holes in the resultant image were filled to make the brain a complete connected component. The resulting pixels are superimposed with the input image for getting the skull removed image. But problem arises when skull and brain are connected together then that method fails to identify the difference between brain and skull.

\section{Proposed Methodology}

Artifacts removal is essential task for normal and abnormality brain tissues identification. Skull elimination is another important step for abnormality segmentation. Sometimes, skull information for particular type of hemorrhage (e.g. Chronic subdural hematoma) lesions segmentation is required due to the needs of distance measurement from skull. Thus in preprocessing method skull removal is not mandatory for some few cases, it is depend on applications. The Figure 1 below shows a flowchart of preprocessing of brain normal and abnormal tissue detection. 


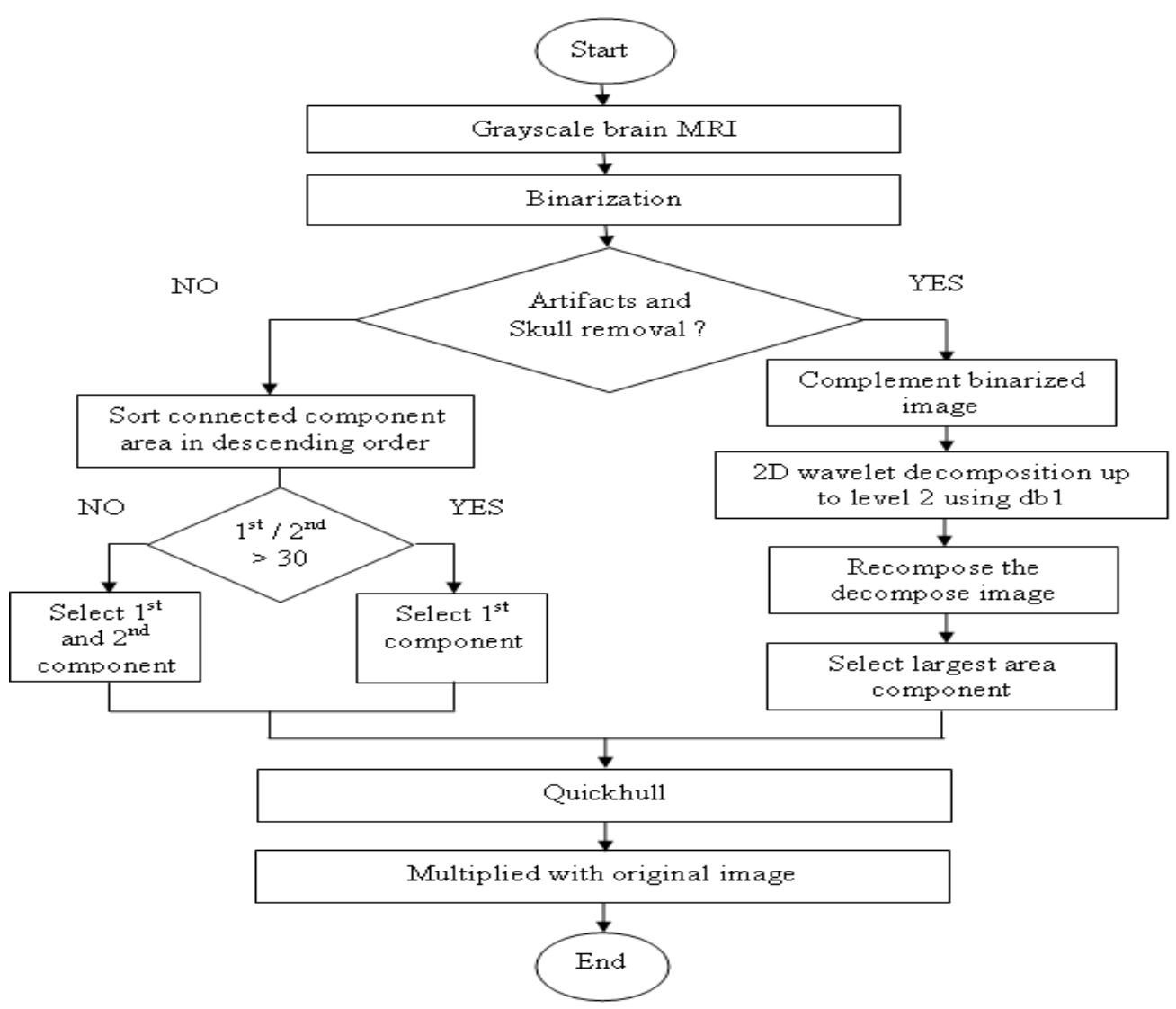

Figure 1. Flow Diagram of Preprocessing for Brain Abnormality and Normal Tissue Detection

An inputted brain MRI first converted into grayscale image and then global threshold intensity has been calculated using standard deviation method. Brain region, skull and many artifacts are converted into white pixels. This binarization step helps to perform connected generation in artifacts removal and wavelet decomposition in artifacts and skull removal. The RGB image has been converted to grayscale image using a weighted sum of the $\mathrm{R}, \mathrm{G}$, and $\mathrm{B}$ components multiplied by a constant. The transformation function is given below

$$
\mathrm{g}(\mathrm{x}, \mathrm{y})=\mathrm{T}[\mathrm{f}(\mathrm{x}, \mathrm{y})]
$$

Here $f(x, y)$ is the input image, $g(x, y)$ is the processed image, and $T$ is an operator on $f$, defined over some neighborhood of $f(x, y)$. In addition, $T$ can operate on a set of input images. The simplest form of $\mathrm{T}$ is when the neighborhood is of size $1 \times 1$ (that is, a single pixel). In this case, $g$ depends only on the value of $f$ at $(x, y)$, and $T$ becomes a gray-level (also called an intensity or mapping) transformation function of the form

$$
\mathrm{s}=\mathrm{T}(\mathrm{r})
$$

For maintaining simplicity in notation, $\mathrm{r}$ and $\mathrm{s}$ are variables denoting, respectively, the gray level of $f(x, y)$ and $g(x, y)$ at any point $(x, y)$. This conversion is followed by image binarization, constituting the preprocessing step and threshold intensity is calculated by standard deviation of the image pixel intensity. To calculate the standard deviation, mean and variance derivation is written below. Mean is defined as the division of the number of samples multiplied by the sum of all data points

$$
\mu=\frac{1}{\mathrm{MN}} \sum_{\mathrm{x}=0}^{\mathrm{M}-1} \sum_{\mathrm{y}=0}^{\mathrm{N}-1} \mathrm{f}(\mathrm{x}, \mathrm{y})
$$


Variance, is denoted as $\mathrm{v}$, equals 1 divided by the number of samples minus one, multiplied by the sum of each data point subtracted by the mean then squared.

$$
\mathrm{v}=\frac{1}{\mathrm{MN}} \sum_{\mathrm{x}=0}^{\mathrm{M}-1} \sum_{\mathrm{y}=0}^{\mathrm{N}-1}(\mathrm{f}(\mathrm{x}, \mathrm{y})-\mu)^{2}
$$

Standard deviation, denoted as $\sigma$, equals the square root of the variance s-squared is written below

$$
\sigma=\sqrt{v}
$$

The obtained standard deviation intensity value is used as threshold intensity to binarize the MR image of brain and is very much helpful for extracting brain portion and differentiating it from to the non-brain portion. MRI of brain has the significant intensity difference between background and the foreground so the use of standard deviation based binarization has been successfully implemented for brain stroke detection purpose.

$$
f 1(x, y)= \begin{cases}1 & \text { if } f(x, y)>0 \\ 0 & \text { if } f(x, y) \leq \sigma\end{cases}
$$

After binary image generation we have an option of artifact and skull removal. Artifacts and skull removal is the one option and only artifacts removal as another options.

Only artifacts removal needs to perform following intermediate steps after performing above steps:

a) Compute the areas of each connected components and descending order of areas are stored in an array. Connected component labeling works by scanning an image, pixel-by-pixel in order to identify connected pixel regions, i.e. regions of adjacent pixels which share the same set of intensity values $V=\{1\}$. The connected components labeling operator scans the image by moving along a row until it comes to a point $p$ (where $p$ denotes the pixel to be labeled at any stage in the scanning process) for which $V=\{1\}$. When this is true, it examines the four neighbors of $p$ which have already been encountered in the scan (i.e. the neighbors (i) to the left of $p$, (ii) above it, and (iii and iv) the two upper diagonal terms). Based on this information, the labeling of $p$ occurs as follows: i) If all four neighbors are 0 , assign a new label to $p$, else ii) if only one neighbor has $V=\{1\}$, assign its label to $p$, else iii) if more than one of the neighbors have $V=\{1\}$, assign one of the labels to $p$ and make a note of the equivalences.

After completing the scan, the equivalent label pairs are sorted into equivalence classes and a unique label is assigned to each class. As a final step, a second scan is made through the image, during which each label is replaced by the label assigned to its equivalence classes.

b) The connected component with the maximum area and second highest area are found out from descending order array. Then ratio between highest and second highest has been performed for the identification of head region (or brain without artifact). i) If the ratio is greater than high (e.g., 30) then keep only highest area and remove all other components. Ratio high (e.g., 30) signifies that the skull are brain are connected together as one component and thus it produce very high ratio between brain and small artifact. That ratio in that case always produces high because artifacts are principally letters which have very less area individually. ii) If ratio is less than 30 (high value taken as 30) then select highest and second highest components and remove others components. If skull and main brain region are disconnected then these two are treated as highest and second highest components. Ratio between skull and main brain never exceeds 30 .

Artifacts and skull removal both need to perform following intermediate steps after performing above steps: 
The negative of an image with gray levels in the range [0, L-1] is obtained by using the negative transformation is given by the expression

$$
s=L-1-r
$$

This complement is helpful for wavelet decomposition because we want to disconnect main brain region from skull where both are connected. Reversing the intensity levels of an image in this manner produces the equivalent of a photographic negative and this type of processing is particularly suited for enhancing white or gray detail fixed in dark regions of an image, especially when the black areas are dominant in size. For binary image complement the algorithm use $\mathrm{f} 2(\mathrm{x}, \mathrm{y})=1-\mathrm{f} 1(\mathrm{x}, \mathrm{y})$ which prepares it for the next step of wavelet decomposition. We begin by defining the wavelet series expansion of function $\mathrm{f} 2(\mathrm{x}) \in \mathrm{L}^{2}(\mathrm{R})$ relative to wavelet $\psi(\mathrm{x})$ and scaling function $\varphi(\mathrm{x}) . \mathrm{f} 2(\mathrm{x})$ can be represented by a scaling function expansion and some number of wavelet function expansions in subspaces $W_{\mathrm{j}_{\mathrm{e}}}, W_{\mathrm{j}_{\mathrm{0}}+1}, W_{\mathrm{j}_{\mathrm{0}+2}} \ldots$. Thus

$$
\mathrm{f} 2(\mathrm{x})=\Sigma_{\mathrm{k}} \mathrm{c}_{\mathrm{j}_{\mathrm{0}}}(\mathrm{k}) \varphi_{\mathrm{j}_{\mathrm{o}}}(\mathrm{x})+\sum_{\mathrm{j}=\mathrm{j}_{\mathrm{o}}}^{\infty} \Sigma_{\mathrm{k}} \mathrm{d}_{\mathrm{j}}(\mathrm{k}) \psi_{\mathrm{j}, \mathrm{k}}(\mathrm{x})
$$

Where $\mathrm{j}_{0}$ is an arbitrary starting scale and the $\mathrm{c}_{\mathrm{j}_{0}}(\mathrm{k})$ and $\mathrm{d}_{\mathrm{j}}(\mathrm{k})$ are relabeled. The $\mathrm{c}_{\mathrm{j}_{\mathrm{o}}}(\mathrm{k})$ normally called approximation or/and scaling coefficients; the $\mathrm{d}_{\mathrm{j}}(\mathrm{k})$ are referred to as detail or/and wavelet coefficients. Thus in the above equation first sum uses scaling function to provides an approximation of $\mathrm{f} 2(\mathrm{x})$ at scale $\mathrm{j}_{0}$. For each higher scale $\mathrm{j} \geq \mathrm{j}_{0}$ in the second sum, a finer resolution function a sum of wavelet is added to the approximation to provide increasing details. If the expansion function forms an orthogonal basis or tight frame, which is often the case, the expansion coefficients are calculated and is shown by the equations below

and

$$
c_{\mathrm{j}_{\mathrm{0}}}(\mathrm{k})=\left\langle\mathrm{f} 2(\mathrm{x}), \varphi_{\mathrm{j}_{\mathrm{d}}}(\mathrm{x})\right\rangle=\int \mathrm{f} 2(\mathrm{x}) \varphi_{\mathrm{j}_{\mathrm{0}}}(\mathrm{x}) \mathrm{dx}
$$

$$
d_{j}(k)=<f 2(x), \psi_{j, k}(x)>=\int f 2(x) \psi_{j, k}(x) d x
$$

Above two coefficients expansion are defined as inner products of function being expanded and the expansion functions being used where $\varphi_{\mathrm{j}_{0}}$ and $\psi_{\mathrm{j}_{\mathrm{j}} \mathrm{k}}$ are the expansion functions; $\mathrm{c}_{\mathrm{j}_{\mathrm{o}}}$ and $\mathrm{d}_{\mathrm{j}}$ are the expansion coefficients. Two dimensional (2-D) scaling function, $\varphi(\mathrm{x}, \mathrm{y})$, which is a product of two 1-D functions and three two dimensional wavelets, $\psi^{\mathrm{H}}(\mathrm{x}, \mathrm{y}), \psi^{\mathrm{V}}(\mathrm{x}, \mathrm{y})$, and $\psi^{\mathrm{D}}(\mathrm{x}, \mathrm{y})$ are required. Excluding the products that produce 1-D results, like $\varphi(\mathrm{x}) \psi(\mathrm{x})$, the four remaining products produce the separable scaling function and separable directionally sensitive wavelets

$$
\begin{gathered}
\varphi(x, y)=\varphi(x) \varphi(y) \\
\psi^{\mathrm{H}}(\mathrm{x}, \mathrm{y})=\psi(\mathrm{x}, \mathrm{y})=\psi(\mathrm{x}) \psi(\mathrm{y}) \\
\psi^{\mathrm{D}}(\mathrm{x}, \mathrm{y})=\psi(\mathrm{y}) \\
\psi(\mathrm{x}) \psi(\mathrm{y})
\end{gathered}
$$

The wavelets measure functional variations, intensity variations for images along different directions: $\psi^{\mathrm{H}}$ measures variations along columns, $\psi^{\mathrm{V}}$ measures variations along rows and $\psi^{\mathrm{D}}$ measures variations along diagonals. The directional sensitivity is a natural consequence of separability in the above equation and it does not increase the computational complexity. The method first defines the scaled and translated basis functions:

$$
\begin{gathered}
\varphi_{\mathrm{j}, \mathrm{m}, \mathrm{n}}(\mathrm{x}, \mathrm{y})=2^{\frac{\mathrm{j}}{2}} \varphi\left(2^{\mathrm{j}} \mathrm{x}-\mathrm{m}, 2^{\mathrm{j}} \mathrm{y}-\mathrm{n}\right) \\
\psi_{\mathrm{j}, \mathrm{m}, \mathrm{n}}^{\mathrm{i}}(\mathrm{x}, \mathrm{y})=2^{\frac{\mathrm{j}}{2}} \psi^{\mathrm{i}}\left(2^{\mathrm{j}} \mathrm{x}-\mathrm{m}, 2^{\mathrm{j}} \mathrm{y}-\mathrm{n}\right), \\
\mathrm{i}=\{\mathrm{H}, \mathrm{V}, \mathrm{D}\}
\end{gathered}
$$


Here index i identifies the directional wavelets. The discrete wavelet transform of image $\mathrm{f} 2(\mathrm{x}, \mathrm{y})$ of size $\mathrm{M} \times \mathrm{N}$ is then denoted by the following equations

$$
\begin{gathered}
W_{\varphi}\left(j_{0}, m, n\right)=\frac{1}{\sqrt{M N}} \sum_{x=0}^{M-1} \sum_{y=0}^{N-1} f 2(x, y) \varphi_{j_{0}, m, n}(x, y) \\
W_{\psi^{i}}(j, m, n)=\frac{1}{\sqrt{M N}} \sum_{x=0}^{M-1} \sum_{y=0}^{N-1} f 2(x, y) \psi_{j, m, n}^{i}(x, y), i=\{H, V, D\}
\end{gathered}
$$

As in the 1-D case, $\mathrm{j}_{0}$ is an arbitrary starting scale and the $\mathrm{W}_{\varphi}\left(\mathrm{j}_{0}, \mathrm{~m}, \mathrm{n}\right)$ coefficients define an approximation $\mathrm{f} 2(\mathrm{x}, \mathrm{y})$ at scale $\mathrm{j}_{0}$. The $W_{\mathrm{\psi}}(\mathrm{j}, \mathrm{m}, \mathrm{n})$ coefficients add horizontal, vertical, and diagonal details for scales $\mathrm{j} \geq \mathrm{j}_{0}$. normally $\mathrm{j}_{0}=0$ and $\mathrm{N}=\mathrm{M}=2^{\mathrm{J}}$ so that $\mathrm{j}=0,1,2, \ldots, \mathrm{J}-1$ and $\mathrm{m}=\mathrm{n}=0,1,2, \ldots, 2^{\mathrm{j}}-1$. Thus $\mathrm{f} 2(\mathrm{x}, \mathrm{y})$ is obtained via the inverse discrete transform as obtained by the equation

$$
\begin{aligned}
\mathrm{f} 2(\mathrm{x}, \mathrm{y})=\frac{1}{\sqrt{\mathrm{MN}}} \sum_{\mathrm{m}} \sum_{\mathrm{n}} \mathrm{w}_{\varphi}\left(\mathrm{j}_{0}, \mathrm{~m}, \mathrm{n}\right) \varphi_{\mathrm{j}_{0}, \mathrm{~m}, \mathrm{n}}(\mathrm{x}, \mathrm{y}) \\
\quad+\frac{1}{\sqrt{\mathrm{MN}}} \sum_{\mathrm{i}=\mathrm{H}, \mathrm{v}, \mathrm{D}} \sum_{\mathrm{j}=\mathrm{j}_{\mathrm{o}}}^{\infty} \sum_{\mathrm{m}} \sum_{\mathrm{n}} \mathrm{w}_{\mathrm{\psi}^{\mathrm{i}}}(\mathrm{j}, \mathrm{m}, \mathrm{n}) \psi_{\mathrm{j}_{\mathrm{j}, \mathrm{m}, \mathrm{n}}^{\mathrm{i}}}^{\mathrm{i}}(\mathrm{x}, \mathrm{y})
\end{aligned}
$$

Select largest connected area of from connected components and remove other components. This largest component is the brain without skull region in MRI.

After performing the artifacts or artifacts and skull removal steps we use some common procedure. The binary image contain white pixels are losses some information in border as well as in side the bounded area. To make it perfect as possible we perform quickhull [9] on white pixels. The quickhull algorithm uses less space than most of the randomized incremental algorithms and executes faster for inputs with non-extreme points. Computation time is less and in addition, quickhull uses merged facets to guarantee that the output is clearly convex. The convex image is now a binary image in which only brain portion is denoted with one and all non-brain portion contains zero. This convex image is multiplied with original image and the resultant image is free of any previously existing artifacts, noise and skull as such removal is critical for brain abnormality detection.

\section{Results and Discussions}

The main title (on the first page) should begin $13 / 16$ inches ( 7 picas) from the top edge of the page, centered, and in Times New Roman 14-point, boldface type.

Proposed method gives suitable results for different MRI of brain images in artifacts removal as well as artifacts and skull removal. The results below have been tested visually and metrically. The procedure of proposed methods has been described above and figures below shows each functional step of the proposed methods. Figure 2 below shows the intermediary results of different intermediate steps of artifacts and skull removal methodology. Brain MRI image (a) is taken as input and converted it to grayscale image for next step binarization. (b) is binarized output using the standard deviation based threshold intensity for input image (a). Maximum of brain region along with artifacts are very clearly visible in binary image. This visibility of maximum brain tissues and artifacts are very useful for next steps. It is clear that the ratio of brain to artifact definitely gives very high (i.e., above 30). The ratio between skull and internal brain tissue will not exceed 30 at any case. So, depending on the ratio between highest to second highest component brain and skull has been selected. (c) is the output after removing artifacts from binary image but some loss of information may possible in border of image as well as inside of image. To reduce the loss of information quickhull method has been used and the result is shown in (d). Pixel wise multiplication has been performed between input image (a) and quickhull image (d) and input image without artifacts is shown in (e). Reference image of 
without artifacts for input image has been shown in (f). Artifact removal by proposed method and reference image shows similar by visually which indicates good preprocessing.

Different intermediate steps are requires for artifacts and skull removal method. The complement of input binarized image is shown (g) that help wavelet decomposition step. (h) is output after applying wavelet decomposition using 'db1' wavelet up to level two. Using wavelet decomposition we reduce the information within the image. If any connection exists in between skull and main brain region then wavelet decomposition helps to disconnect between that connections. Due to the reduction of size in wavelet decomposition we recompose and resize the image in to the initial size. The recomplemented and recomposed image has been shown in (i). Selection of largest area as brain is easier as we disconnect the skull and main brain and largest area has been shown in (j). Some losses of information in (j) and to recover this loss quickhull method applied on (j). Applying quickhull generated correct result has been shown in (k). Then (l) is brain image without any artifacts and skull using the pixel wise multiplication between input image and convex image. Reference binary image of artifacts and skull removal for input (a) has been shown in (m). In visual prospect both proposed method result and reference image are almost similar.

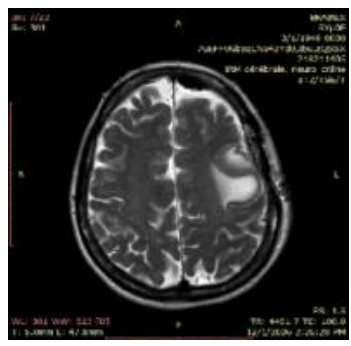

(a) Input Brain MRI

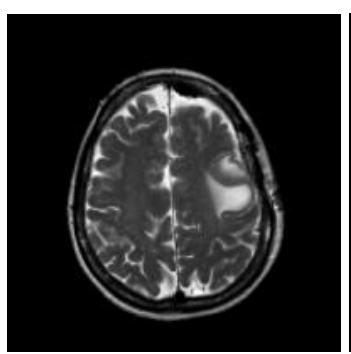

(e) Without Artifacts

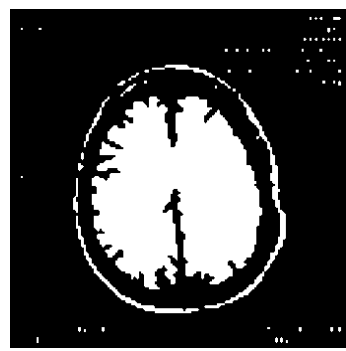

(i) Re-complement

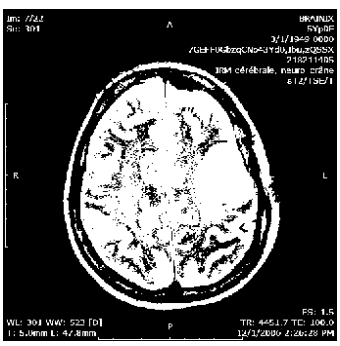

(b) Binarized Image
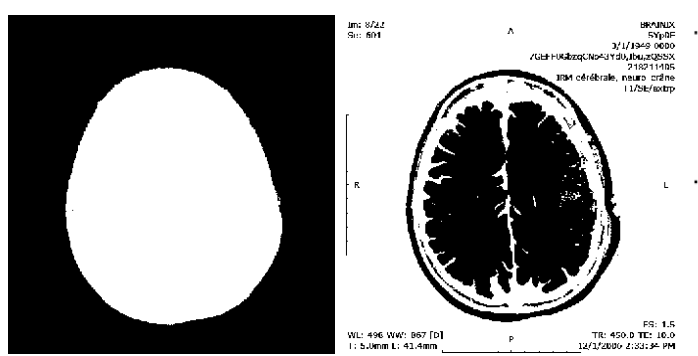

(d) Applying Quickhull
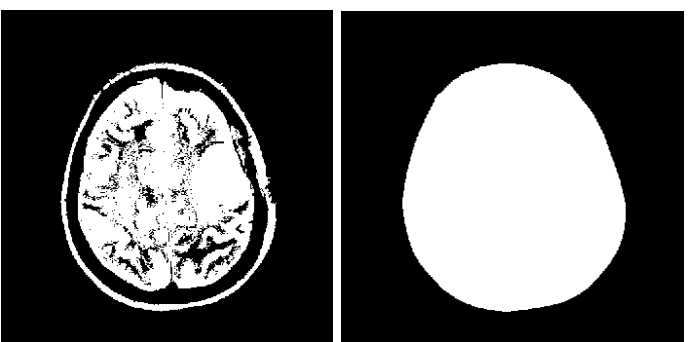

(c) Without Artifact

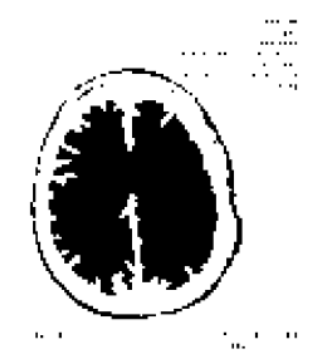

(f) Reference (g) Complement of Input (h) Wavelet Decomposed
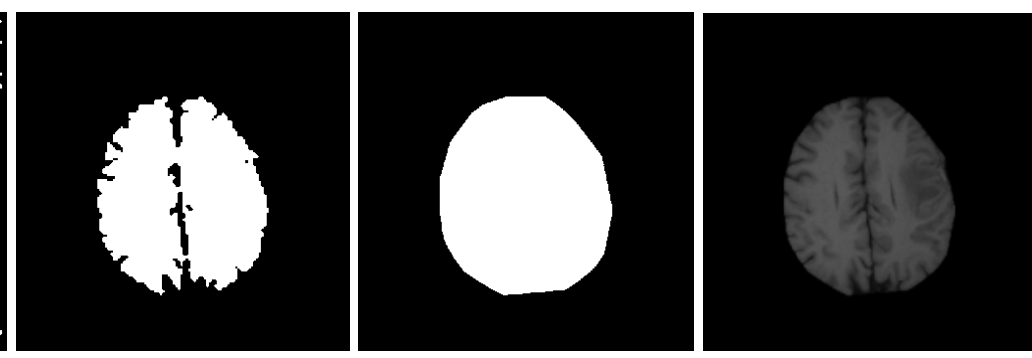

(j) Brain Region

(k) Applying Quickhull

(I) Without Artifacts 


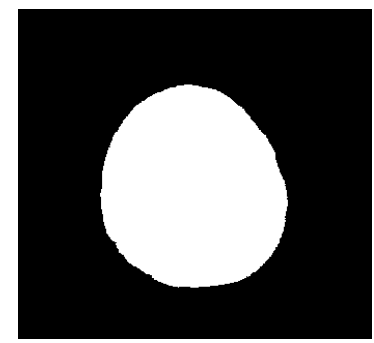

(m) Reference Image for Removed Artifacts and Skull

Figure 2. Output of Different Intermediate Steps of Artifacts and Skull Removal Methodology (b) Is the Binarized Output for Input Image (a), (c) Is the Output after Artifacts Removal, (d) Is Convex Hull Output on Image (c),

(e) Is Input Image without Artifacts, (f) Is Reference Image of without Artifacts for Input Image (a), (g) Is Complement of Inputted Binarized Image (b), (h) Is Output after Applying Wavelet Decomposition, (i) Is ReComplement and Recomposed Result on Image (h), (j) Is the Output after Removing Skull Region, (k) Is Result after Applying Convex Hull to Generate Correct Result, (I) Is Brain Image without Any Artifacts and Skull, and $(m)$ Is Reference Binary Image of Artifacts and Skull Removal for Input

Artifacts removal technique is able to remove the artifacts if any artifacts presents in the brain MRI. Proposed method is tested on large dataset and produce excellent results except connected artifact with the original brain portion image. Proposed technique is very helpful in the sense of brain tissues detection. Artifacts and skull removal technique is also remove skull and artifacts for different images. The correct elimination of skull and artifacts will reduce false detection in abnormal tissues detection. Measure the performance by visually may be biased. Thus some performance evaluation metrics are used to evaluate the error and accuracy with respect to the reference image.

The accuracy is used to evaluate the performance of the proposed methods are the Relative area Error (RE), Kappa Index (KI), Jacard Index (JI), Correct Detection Ratio (CDR) and False Detection Ratio (FDR). A critical problem faced in performance evaluation of artifacts and skull removal method is the lack of a gold standards. Here we use ground truth suggested by radiologist for the comparison with the automated method and measures their performance with the help of RE, KI, JI, CDR, FDR [10,11]. Let AS and MS denote the area of the automatically segmented (AS) and manually segmented (MS) regions of the MR brain images. The RE for segmented region can be calculated as division of difference between 'AS' and 'MS' to 'MS'. RE in percentage is given below:

$$
R E=\frac{\| A S-M S \mid}{M S} * 100 \%
$$

Which measure the relative area difference with respect to the manual ground truth segmented, in other words we can say how much it differ from actual results. A method could be better when RE value is less, so the best method would be minimum value of RE. The KI between two areas is calculated by the following equation:

$$
\mathrm{KI}(A S, M S)=\frac{(2|T P|)}{(|A S|+|M S|)} * 100 \%
$$

Where TP is the intersection of pixels between MS and AS, called true positive, and it determines correctness of method. KI determine correctness with respect to the automated and manual segmentation. $\mathrm{KI}$ is also represented as similarity index, which is sensitive to both differences in size and location. A method could be better when KI value is more, so the best method would be maximum value of KI. The JI between two areas is represented as follow: 


$$
J \mathrm{I}(A S, M S)=\frac{\|T P\|}{\|T P+F N+F P\|} * 100 \%
$$

Where false positive FP = AS - TP determine how much AS deviated from true positive and false negative FN = MS - TP determine how much MS deviated from true positive. This metric is further susceptible to variation since both denominator and numerator change with rising or falling overlap. CDR or sensitivity is defined by the following equation:

$$
\mathrm{CDR}=\frac{\| \text { ASnMS } \mid}{\text { MS }} * 100 \%
$$

FDR is determine by

$$
\mathrm{FDR}=\frac{\|\mathrm{AS}-\mathrm{TP}\|}{\text { MS }} * 100 \%
$$

Higher value of correct detection ratio and lower value of false detection ratio means the good results. A method could be better when JI, and CDR value is more and less value of FDR, so the best method would be maximum value of JI, and CDR and minimum value of FDR. Different performance metric (AS, MS, RE, TP, FP, FN, KI, JI, CDR, FDR) has been shown in Table below for 10 images $[12,13,14]$ for evaluation errors and accuracy of our results. We have tested our method on 450 images from different dataset.

Segmented area of brain and brain without skull using proposed method with $341 \times$ 341 image size has been shown in Table 1. Area of reference segmentation and intersection between reference and proposed method are also displayed in Table 1. Intersection pixels determines the exact number of pixels matches between automated segmented and manual (reference) segmented. The intersection nearer to the automated and manual segmentation indicates good segmentation and its intersection value has been shown in Table 1 .

Table 1. Area of without Artifacts and Brain without Skull Using Proposed and Manual Segmentation with their Intersection

\begin{tabular}{|c|c|c|c|c|c|c|}
\hline \multirow{2}{*}{$\begin{array}{c}\text { Image } \\
\text { sequence }\end{array}$} & \multicolumn{3}{|c|}{ Without Artifacts } & \multicolumn{3}{c|}{ Brain Without Skull } \\
\cline { 2 - 7 } & $\begin{array}{c}\text { Automate } \\
\mathrm{d}\end{array}$ & $\begin{array}{c}\text { Manua } \\
1\end{array}$ & Intersection & $\begin{array}{c}\text { Automate } \\
\mathrm{d}\end{array}$ & $\begin{array}{c}\text { Manu } \\
\text { al }\end{array}$ & $\begin{array}{c}\text { Intersectio } \\
\mathrm{n}\end{array}$ \\
\hline 1 & 44246 & 45148 & 44232 & 30423 & 31062 & 30375 \\
\hline 2 & 43672 & 44484 & 43649 & 28210 & 29125 & 28189 \\
\hline 3 & 44135 & 44492 & 44125 & 30156 & 30483 & 30126 \\
\hline 4 & 43581 & 44007 & 43389 & 29479 & 30282 & 29442 \\
\hline 5 & 45278 & 45392 & 45198 & 30281 & 30933 & 30241 \\
\hline 6 & 44288 & 45183 & 44206 & 29940 & 30426 & 29937 \\
\hline 7 & 43218 & 44090 & 43183 & 28943 & 29471 & 28941 \\
\hline 8 & 43826 & 44164 & 43820 & 29781 & 30282 & 29768 \\
\hline 9 & 43002 & 44083 & 42960 & 29162 & 30001 & 29153 \\
\hline 10 & 43539 & 44006 & 43502 & 29872 & 31206 & 29866 \\
\hline
\end{tabular}

In medical imaging low error is required as much as possible because increased error reflects wrong diagnosis. Removing artifacts and skull by keeping all necessary information (soft tissues of brain) is the key goal of preprocessing. The RE and FDR for brain with skull (artifact removal) and without skull (artifacts and skull removal) are shown in Table 2 below. 
Table 2. RE and FDR Performance Metric

\begin{tabular}{|c|c|c|c|c|}
\hline \multirow{2}{*}{$\begin{array}{c}\text { Image } \\
\text { sequence }\end{array}$} & \multicolumn{2}{|c|}{ RE } & \multicolumn{2}{c|}{ FDR } \\
\cline { 2 - 5 } & $\begin{array}{c}\text { Without } \\
\text { Artifacts }\end{array}$ & $\begin{array}{c}\text { Brain Without } \\
\text { Skull }\end{array}$ & $\begin{array}{c}\text { Without } \\
\text { Artifacts }\end{array}$ & $\begin{array}{c}\text { Brain Without } \\
\text { Skull }\end{array}$ \\
\hline 1 & 1.99 & 2.05 & 0.03 & 0.15 \\
\hline 2 & 1.82 & 3.14 & 0.05 & 0.07 \\
\hline 3 & 0.80 & 1.07 & 0.02 & 0.09 \\
\hline 4 & 0.96 & 2.65 & 0.43 & 0.12 \\
\hline 5 & 0.25 & 2.10 & 0.17 & 0.12 \\
\hline 6 & 1.98 & 1.59 & 0.18 & 0.01 \\
\hline 7 & 1.97 & 1.79 & 0.07 & 0.01 \\
\hline 8 & 0.76 & 1.65 & 0.01 & 0.04 \\
\hline 9 & 2.45 & 2.79 & 0.09 & 0.03 \\
\hline 10 & 1.06 & 4.27 & 0.08 & 0.02 \\
\hline
\end{tabular}

From the above table all RE values in without artifacts are less than 3 and RE values in brain without skull are less than 5. FDR values for both without artifacts and brain without skull are less than 1. RE less means less over and under segmentation and FDR less means less false detection. In our preprocessing FDR and RE both are very less values which indicate a good preprocessing. The column chart representation of RE and FDR values for 10 images are shown in Figure 3 below.

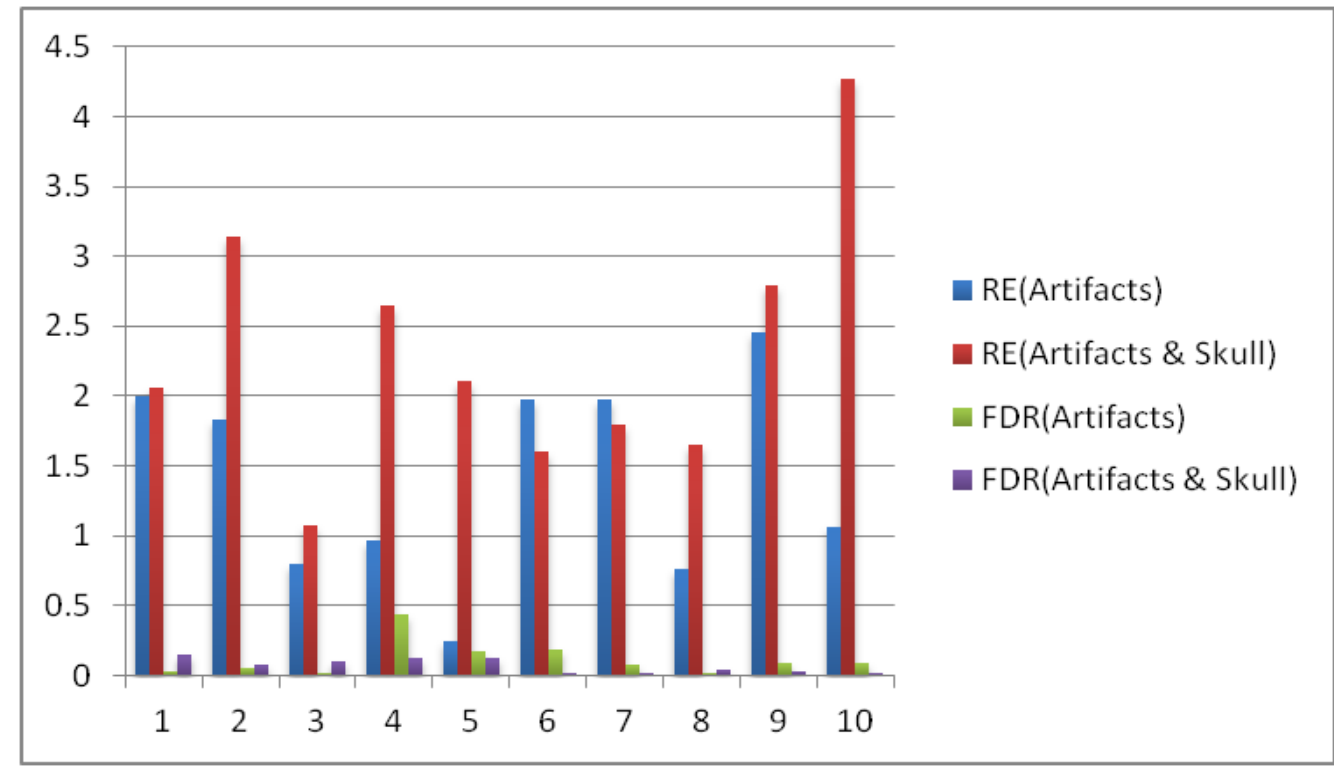

Figure 3. Column Chart Representation of RE and FDR Metrics of Preprocessing Method

From column chart it is clear that maximum $\mathrm{RE}$ value is less than 3 and maximum FDR value is less than 2. Thus proposed preprocessing method is useful for brain abnormality detection as it generates very low error.

The usefulness of preprocessing method also depends on correct segmentation or preprocessing. The values of different accuracy metrics KI, JI and CDR for preprocessing have been shown in Table 3 below. 
Table 3. KI, JI and CDR Performance Metric

\begin{tabular}{|c|c|c|c|c|c|c|}
\hline \multirow{2}{*}{$\begin{array}{c}\text { Image } \\
\text { sequence }\end{array}$} & \multicolumn{2}{|c|}{ KI } & \multicolumn{2}{c|}{ JI } & \multicolumn{2}{c|}{ CDR } \\
\cline { 2 - 7 } & $\begin{array}{c}\text { Without } \\
\text { Artifacts }\end{array}$ & $\begin{array}{c}\text { Brain } \\
\text { Without } \\
\text { Skull }\end{array}$ & $\begin{array}{c}\text { Without } \\
\text { Artifacts }\end{array}$ & $\begin{array}{c}\text { Brain } \\
\text { Without } \\
\text { Skull }\end{array}$ & $\begin{array}{c}\text { Without } \\
\text { Artifacts }\end{array}$ & $\begin{array}{c}\text { Brain } \\
\text { Without } \\
\text { Skull }\end{array}$ \\
\hline 1 & 98.96 & 98.80 & 97.94 & 97.64 & 97.97 & 97.79 \\
\hline 2 & 99.03 & 98.33 & 98.07 & 96.72 & 98.12 & 96.78 \\
\hline 3 & 99.57 & 99.36 & 99.15 & 98.73 & 99.18 & 98.82 \\
\hline 4 & 99.08 & 98.53 & 98.16 & 97.11 & 98.60 & 97.22 \\
\hline 5 & 99.68 & 98.80 & 99.39 & 97.64 & 99.57 & 97.76 \\
\hline 6 & 98.82 & 99.18 & 97.66 & 98.38 & 97.83 & 98.39 \\
\hline 7 & 98.92 & 99.09 & 97.86 & 98.19 & 97.94 & 98.20 \\
\hline 8 & 99.60 & 99.12 & 99.21 & 98.26 & 99.22 & 98.30 \\
\hline 9 & 98.66 & 98.55 & 97.36 & 97.14 & 97.45 & 97.17 \\
\hline 10 & 99.38 & 97.80 & 98.77 & 95.69 & 98.85 & 95.71 \\
\hline
\end{tabular}

Both without artifacts and brain without skull generates KI value greater than $98 \%$. Thus this high KI indicates correctness with respect to the automated and manual segmentation. As KI is sensitive to both in size and location thus with respect to similarity measurement proposed preprocessing generates very good accuracy. JI values for both methods are greater than $97 \%$ that indicates good accuracy. CDR values for both methods are greater than $97 \%$ that signifies preprocessing technique correctly segment the desire region of brain. The column chart representation of KI, JI and CDR values for 10 images are shown in Figure 4 below.

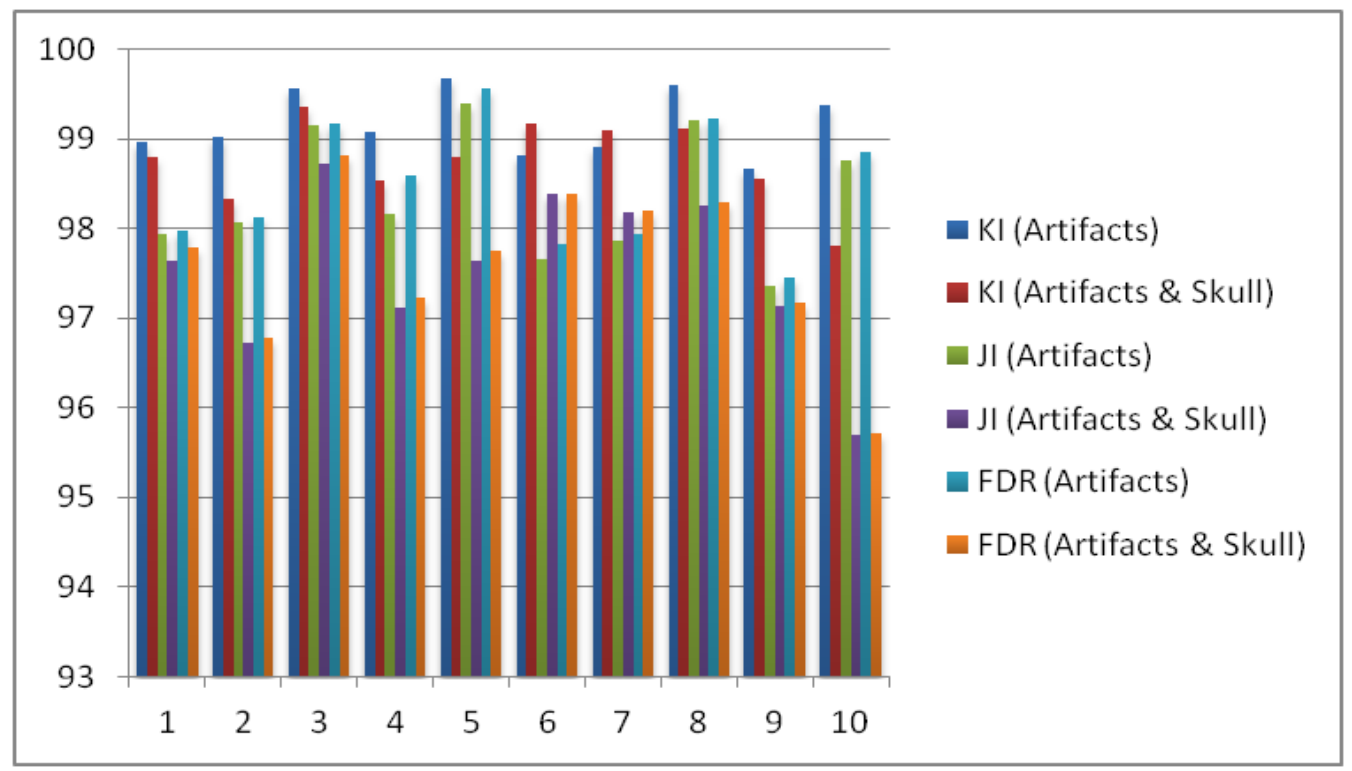

Figure 4. Column Chart Representation of KI, JI and CDR Metrics of Preprocessing Method

Proposed preprocessing technique generates very high accuracy which is clearly visible from column chart. High accuracy means correct identification and it can be used as preprocessing. In other context high accuracy increases the chances of good postprocessing because good abnormality detection technique depends on good prepreprocessing. The average value of performance evaluation metric RE, FDR, KI, JI, and CDR for 10 images is shown in Table 4 below. 


\section{Table 4. Average Value of Error and Accuracy Metric}

\begin{tabular}{|c|c|c|c|c|c|}
\hline & RE & FDR & KI & JI & CDR \\
\hline Artifacts & 1.41 & 0.12 & 99.17 & 98.36 & 98.47 \\
\hline Artifacts and Skull & 2.31 & 0.07 & 98.76 & 97.55 & 97.61 \\
\hline
\end{tabular}

The average value of different error metrics is very less in both artifacts removal and artifacts and skull removal techniques. Average values of different accuracy metrics are also very high for both artifacts removal and artifacts and skull removal techniques. Thus a preprocessing with very high accuracy and very low error metric represents good preprocessing techniques.

\section{Conclusion}

In this paper, a fully automatic method for artifacts and skull removal of brain MR images using computational geometry, wavelet decomposition and thresholding as intermediate steps has been described. Thresholding using standard deviation method is key intermediate step to correct preprocessing of MR images. The proposed preprocessing method has high accuracy and low error rate for different MR images. This preprocessing is used to reduction of false detection thus it increase the diagnosis quality of disease from MRI of brain by computer system. Thus reprocessing with low error and high accuracy does not hamper the post-processing of intelligence system. The proposed method is very useful and important preprocessing for correct automated detection of brain abnormalities. This preprocessing method is used in abnormalities detection, segmentation and classification and tissues identification in this research.

\section{References}

[1] A.S. Suresh, W. Zheng, W.L.C. Michael, and V. Zagorodnon, "Skull Stripping using Graph Cuts," Neuroimage, vol. 49, (2009), pp. 225-239.

[2] G.J. Park and C. Lee, "Skull Stripping Based on Region Growing for Magnetic Resonance Images," Neuroimage, volume 47, no. 4, (2009), pp. 1394- 1407.

[3] K. Somasundaram and T. Kalaiselvi, "Fully Automatic Brain Extraction Algorithm for Axial T2Weighted Magnetic Resonance Images", Computers in Biology and Medicine, vol. 40, no. 10, (2010), pp. 811-822.

[4] K. Somasundaram, R. Siva Shankar, "Skull Stripping of MRI Using Clustering and 2D Region Growing Method," Image Processing NCIMP, (2010), pp.1-12.

[5] W. Speier, J. E. Iglesias, L. El-Kara, Z. Tu and C. Arnold, "Robust Skull Stripping of Clinical Glioblastoma Multiforme Data”, MICCAI 2011, Part III, Springer LNCS 6893, (2011), pp. 659-666.

[6] A.G. R. Balan, A.J.M.T. Marcela, X. Ribeiro, P. M. A. Marques and C. Traina, "Smart Histogram Analysis Applied To The Skull-Stripping Problem In T1-Weighted MRI", Computers in Biology and Medicine, 42, (2012), pp.509-522.

[7] J Kleesiek, G. Urban, A. Hubert, D. Schwarz, K. Maier-Hein, M. Bendszus, A. Biller, "Deep MRI brain extraction: A 3D convolutional neural network for skull stripping", Neuroimage, (2016), pp. 1-13.

[8] C. C. Benson, V. L. Lajish, K. Rajamani, "A Novel Skull Stripping and Enhancement Algorithm for the Improved Brain Tumor Segmentation using Mathematical Morphology", I.J. Image, Graphics and Signal Processing, vol. 7, (2016), pp. 59-66.

[9] C. Bradford Barber, David P. Dobkin, HannuHuhdanpaa, "The Quickhull Algorithm for Convex Hulls", ACM Transactions on Mathematical Software, vol. 22, no. 4, (1996), pp. 469-483

[10] S. Roy, D. Bhattacharyya, S.K. Bandyopadhyay, T. Kim, "An Effective Method for Computerized Prediction and Segmentation of Multiple Sclerosis Lesions in Brain MRI", Computer Methods and Programs in Biomedicine, ELSEVIER, vol. 140, C, (2017), pp. 307-320.

[11] S. Roy, D. Bhattacharyya, S.K. Bandyopadhyay, T. Kim, "An Improved Brain MR Image Binarization Method as a Preprocessing for Abnormality Detection and Features Extraction," Frontiers of Computer Science, Springer pp. 1-11, 2017. DOI: 10.1007/s11704-016-5129-y. (In press)

[12] Whole Brain Atlas: MR brain image available online (2014): http://www.med.harvad.edu/AANLIB/home.html

[13] BrainWeb: Simulated Brain MR brain image dataset available online (2014): http://brainweb.bic.mni.mcgill.ca/brainweb/ 
[14] The EASI MRI Home: MR brain image available online (2013), http://www.easidemographics.com/ cgibin/dbmri.asp

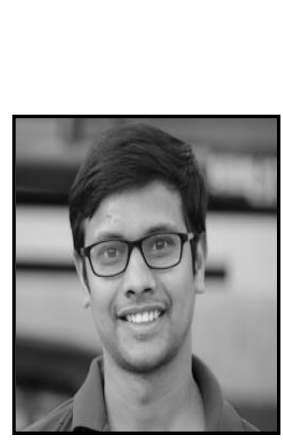

\begin{abstract}
Authors
Sudipta Roy, has submitted his Ph.D.(Comp. Sc. \& Engg.) thesis in the field of image processing in the department of Computer Science and Engineering, University of Calcutta, Kolkata, India on 2017. He received his B.Tech and M.Tech degree in the department of Computer Science \& Engineering from University of Calcutta, in 2011 and 2013. He received his B.Sc (Physics Hons) from Burdwan University in 2008. He is an author of more than twenty publications in National and International Journals and conferences including IEEE, Springer, Elsevier and many others. He is an author of one book and filed one US patient in the field of medical image processing. He have served as a reviewer of many international journals including Springer, Elsevier, IET and many others, and international conferences. He is International Advisory Committee member of many conferences. He has 4 years of experience in Teaching, and Research. His fields of interest are Biomedical Image Analysis, Image Processing, Steganography, Artificial Intelligence, Big data Analysis, Machine Learning and Big data Technologies. Currently, he is working as an Assistant Professor in the department of Computer Science and Engineering, U. V. Patel College of Engineering, Ganpat University.
\end{abstract}

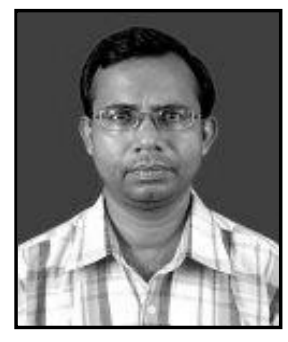

Debnath Bhattacharyya, received Ph.D. (Tech, Computer Science and Engineering) from University of Calcutta, and M.Tech. (Computer Science and Engineering) from West Bengal University of Technology, Kolkata. Currently, he is associated as a Professor with Department of Computer Science and Engineering, Vignan's Institute of Information Technology, Visakhapatnam 530049, AP, India. He has 18 years of experience in Teaching, and Research. His research interests include Bio Informatics, Image Processing and Pattern Recognition. He has published 145 Research Papers in International Journals and Conferences and 4 Text Books for Computer Science.

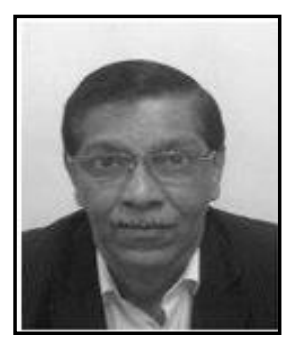

Samir Kumar Bandyopadhyay, B.E., M. Tech., Ph. D (Computer Science \& Engineering), C. Engg., D. Engg., FIE, FIETE, Sr. Member IEEE, currently, Professor of Computer Science \& Engineering, University of Calcutta, Kolkata, India. Visiting Faculty, Dept. of Comp. Sc., Southern Illinois University, USA, MIT, California Institute of Technology, etc. He is Chairman, Science \& Engineering Research Support Society (SERSC, Indian Part), Fellow of Computer Society of India, Sectional President of ICT of Indian Science Congress Association, 2008-2009, Senior Member of IEEE, Member of ACM, Fellow of Institution of Engineers (India), Fellow of Institution of Electronics \& Tele Communication Engineering, India, Reviewer of International Journals IEEE Trans on Neural Networks, ACM, Springer Publications. His research interests include Bio-medical Engg, Mobile Computing, Pattern Recognition, 
Graph Theory, Software Engg., etc. He has published 300 Research papers in International \& Indian Journals and 5 leading text books for Computer Science and Engineering. He is the former Registrar of University of Calcutta and West Bengal University of Technology, Kolkata, and former Acting-VC, West Bengal University of Technology, Kolkata, India.

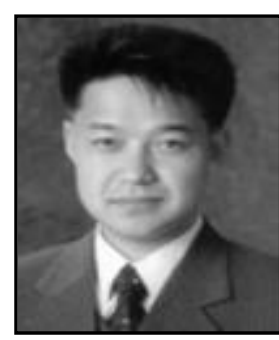

Tai-hoon Kim is, received B.E., and M.E., degrees from Sungkyunkwan University in Korea and and Ph.D. degrees from University of Bristol in UK and University of Tasmania in Australia. Now he is working for Department of Convergence Security, Sungshin W. University, Korea. His main research areas are security engineering for IT products, IT systems, development processes, and operational environments. He published 300 Research Papers in International Journals and Conferences. He is editor of Elsevier, Springer, and many more journals. 